\title{
Positive Remodeling - a Major Feature of Vulnerability in Patients with Non- Obstructive Coronary Artery Disease
}

\author{
Evelin Szabó, Diana Opincariu, András Mester, Alexandra Stănescu, Nora Rat, Theodora Benedek \\ Center of Advanced Research in Multimodality Cardiac Imaging, Cardio Med Medical Center, Târgu Mureș, Romania
}

\section{CORRESPONDENCE}

Diana Opincariu

Str. 22 Decembrie 1989 nr. 76

540124 Târgu Mureș, Romania

Tel: +40 265217333

E-mail: diana.opincariu@yahoo.ro

\section{ARTICLE HISTORY}

Received: November 27, 2020

Accepted: February 8, 2021

Published online February 20, 2021
Evelin Szabó • Str. 22 Decembrie 1989 nr. 76, 540124 Târgu Mureș, Romania. Tel: +40 265217 333, E-mail: szaboevelin22@yahoo.com

András Mester • Str. 22 Decembrie 1989 nr. 76 , 540124 Târgu Mureș, Romania. Tel: +40 265217333 , E-mail: andras.mester@yahoo.com

Alexandra Stănescu • Str. 22 Decembrie 1989 nr. 76 540124 Târgu Mureș, Romania. Tel: +40 265217333 , E-mail: alexandrastanescu90@gmail.com

Nora Rat • Str. 22 Decembrie 1989 nr. 76, 540124 Târgu Mureș, Romania. Tel: +40 265217 333, E-mail: ratnora@gmail.com

Theodora Benedek • Str. 22 Decembrie 1989 nr. 76, 540124 Târgu Mureș, Romania. Tel: +40 265217 333, E-mail: theodora.benedek@gmail.com

\begin{abstract}
The most common cause of acute coronary syndrome is thrombosis of an atheromatous plaque. Positive remodeling is the compensatory dilatation of the plaque-containing section of the vessel wall. Plaques are most commonly characterized as vulnerable when possessing some of the following features: fibrous cap thickness $<65 \mu \mathrm{m}$, large necrotic lipid core, high degrees of inflammatory infiltrates, positive remodeling, intraplaque hemorrhage, or neoangiogenesis. The presence of these plaque features is associated with high cardiovascular risk. In the initial stage of vasculopathy, due to positive remodeling, lumen reduction is not typical; it only develops in the advanced phase of the disease, due to which, based on a lumenogram, the vascular system may appear intact. Therefore, coronary angiography can easily miss the diagnosis or underestimate its extent, since it does not inform us of the composition of the arterial wall, because the contrast agent is just filling the vessel lumen. Coronary CT angiography may fill this diagnostic gap, since changes of the vessel wall can directly be visualized. To increase diagnostic accuracy, invasive coronary angiography can be completed by intravascular ultrasound and optical coherence tomography.
\end{abstract}

Keywords: vulnerable plaque, acute coronary syndrome, cardiac computed tomography angiography, optical coherence tomography, virtual histology intravascular ultrasound

\section{INTRODUCTION}

The most common cause of acute coronary syndrome is thrombosis of an atheromatous plaque. In the past, studies have suggested that the primary cause of acute coronary syndromes is coronary stenosis caused by the thickening of the plaque, which decreases blood flow, leading to ischemia. But recent data suggest that the stenois is due to rupture or erosion of a vulnerable plaque, while calcified plaques are less common. To avoid advanced coronary disease, it is important to recognize in time the features that predispose to plaque vulnerability. ${ }^{1,2}$

Plaques are most commonly characterized as vulnerable when possessing some of the following features: fibrous cap thickness $<65 \mu \mathrm{m}$, large necrotic lipid 
core, high degrees of inflammatory infiltrates, positive remodeling, intraplaque hemorrhage, or neoangiogenesis. ${ }^{3,4}$

Positive remodeling represents the compensatory dilatation of the plaque-containing section of the vessel wall, so that the lumen diameter does not change, no significant narrowing develops, and the patient does not have any symptoms. The presence of these plaque features is associated with high cardiovascular risk. ${ }^{5}$

The mechanism of arterial remodeling was first described in 1953 by Crawford and Levene, who stated that "ordinary atheromatous plaques do not project into the lumen but lie in a depression in the media, which may bulge outwards." Glagov et al. showed a significant positive correlation between the area of the internal elastic lamina and that of the plaque, pointing out that only the expansion of the external elastic membrane could be observed until the lesion has reached $40 \%$ area stenosis. ${ }^{7}$ In the early 90 s, in vivo studies demonstrated through intravascular ultrasound (IVUS) a similar correlation between early plaque accumulation and compensatory enlargement of vessel size. ${ }^{8}$ Further histologic and IVUS studies demonstrated that arterial remodeling could be bidirectional. "Positive remodeling", as observed by Glagov et al., describes an expansion in the external elastic membrane area and "negative remodeling" describes a reduction of the external elastic membrane area at the lesion site. ${ }^{4}$

A histopathological study conducted by Varnava et al. in the early 2000s sought to determine the association between plaque composition and coronary remodeling. ${ }^{9} \mathrm{By}$ this time, several studies have discussed that the outward dilation of the vessel wall is not an advantage but rather dangerous, as marked compensatory remodeling can make the plaque vulnerable, ${ }^{7,10}$ while vessel shrinkage may appear more stable. ${ }^{11,12}$

Varnava et al. have shown that coronary artery plaques that have undergone positive remodeling have a significantly larger lipid core, higher macrophage numbers, and eccentric distribution that may undergo a more regionalized restructuring of the vessel wall, allowing outward expansion. The vessel narrows where it has a low index of vulnerability to plaque rupture (low lipid content and low macrophage number), and it is associated with a more circumferential distribution of the plaque and adventitial thickening. ${ }^{9}$

\section{DEFINITIONS OF REMODELING}

The definition of remodeling varies among studies. ${ }^{13} \mathrm{Re}$ modeling can be quantified using the remodeling index (RI). RI was defined as the external elastic membrane (EEM) at the minimal lumen area (MLA) divided by the average of the proximal and distal reference external elastic membrane areas at the cross sectional areas (CSA). ${ }^{10,14-19}$ Positive RI cut points vary in studies between 1.00 and1.05, while a negative RI is generally considered $<0.88$, and an intermediate RI between 0.88 and $1.00 . .^{14,17}$

\section{DIAGNOSTIC METHODS}

Invasive coronary angiography (ICA) has been the only available method for to imaging assessment of coronary arteries, and it is still the gold standard. ${ }^{20}$ In the initial stage of vasculopathy, due to positive remodeling, lumen reduction is not typical. It only develops in the advanced phase of the disease, due to which, based on a lumenogram, the vascular system may appear intact. ICA can easily miss the diagnosis or underestimate its extent, and it does not provide information regarding the composition of the arterial wall, since the contrast agent is just filling the vessel lumen. ${ }^{20}$

\section{Coronary CT Angiography}

A frequent feature associated with plaque rupture is the thin-cap fibroatheroma (TCFA), which is characterized by a necrotic core with an overlying thin-ruptured fibrous cap (measuring less than $65 \mu \mathrm{m}$ in thickness) infiltrated by macrophages and lymphocytes. ${ }^{21,22}$ TCFA is frequently associated with expansive remodeling. ${ }^{20}$

These changes cannot be assessed by ICA because the vessel wall is not detectable; only the lumen becomes visible, which may appear normal.

Coronary CT angiography (CCTA) may fill this diagnostic gap, since changes of the vessel wall can be directly visualized using this modern technique. ${ }^{20}$ CCTA is the preferred test in the exclusion of coronary artery disease in patients with a lower range of clinical likelihood of coronary artery disease, no previous diagnosis of coronary artery disease, and characteristics associated with a high likelihood of good image quality. ${ }^{23,24}$ Noninvasive functional imaging or anatomical imaging using CCTA is recommended in patients with suspected or newly diagnosed coronary artery disease and may be used as the initial test to rule out or establish the diagnosis. ${ }^{24-27}$

Traditionally, plaque analysis is done visually, subjectively, but diagnostic accuracy can be significantly improved by using automated analysis software. In CCTA imaging, in addition to the degree of lumen narrowing and the calcium content of atherosclerotic plaques, lesion characteristics, such as plaque morphology, plaque load, and plaque remodeling can also be assessed more accurately. 
In a follow-up study, Motoyama et al. analyzed previous CCTA recordings of individuals with acute coronary syndrome. The risk of acute coronary syndrome was higher in individuals with positive remodeling and low-attenuation plaque..$^{28,29,30}$

Another study from 2020, which included 1,005 patients, demonstrates that adverse plaque characteristics (defined as the presence of positive remodeling, low-attenuation plaque, or spotty calcification) are associated with a higher cardiovascular risk, at the same time being linked with an accelerated progression of new adverse plaque characteristics. ${ }^{31}$

The clinical utility of CCTA was revealed by post-hoc analyses of the SCOT-HEART data, a study that investigated the extent of adverse coronary artery plaque characteristics on CCTA and their association with subsequent clinical outcomes. The presence of positive remodeling or low-attenuation plaque (defined as adverse plaque) was associated with a greater risk of death from coronary heart disease and non-fatal myocardial infarction in non-obstructive and obstructive coronary artery disease. Importantly, the study demonstrated that the characterization of plaque morphology had a prognostic value for high-risk vulnerable plaques, and it may help risk stratification and guide the intensity of therapy. ${ }^{32,33}$

\section{Intravascular Ultrasound}

The development of modern imaging technologies, such as intravascular ultrasound (IVUS), has made it possible to study coronary arterial remodeling in vivo. ${ }^{34}$

IVUS allows the visualization of the entire vessel wall structure and morphology. The use of IVUS allows the calculation of eccentricity and symmetry indices and the evaluation of subsequent vascular lumen remodeling. ${ }^{35,36}$

Early in vivo studies of human coronary arteries using IVUS imaging found a correlation between the area of the atheroma and that of the EEM ${ }^{8}$

Positive remodeling has been observed in the coronary arteries in acute coronary syndromes. It is generally associated with vulnerable plaques and high thrombus burden, leading to distal embolization. ${ }^{9,10}$

A small clinical study examined non-culprit, non-obstructive lesions, using IVUS to assess the implications of plaque composition and morphology in the mechanism of vascular remodeling. In lesions showing positive remodeling, the size of the lipid core was significantly larger, whereas the fibrotic burden was significantly and inversely correlated with the remodeling index. Positively remodeled lesions have a higher risk phenotype to rupture, as they contain a thin-cap fibroatheroma. In contrast, lesions with negative remodeling were found to have intima thickening, a more stable phenotype. ${ }^{16}$

The European Collaborative Project on Inflammation and Vascular Wall Remodeling in Atherosclerosis - Intravascular Ultrasound (ATHEROREMO-IVUS) study was designed to investigate the associations between genetic profile, coronary atherosclerosis phenotype, and vulnerability determined by IVUS. The study observed that single-nucleotide polymorphism Rs6932 is associated with remodeling and is near the SLC22A25 gene, in addition to being strongly associated with IVUS-derived vulnerability indicators in or near eight different genes. ${ }^{37}$

At the same time, the AtheroRemo-IVUS study pointed out the significant association between the necrotic core in the imaged coronary segment and major acute cardiovascular events, suggesting that imaging biomarkers are useful clinical tools in assessing the future risk of major cardiovascular complications in patients with coronary artery disease. ${ }^{38}$

Coronary slow flow following stent implantation is an important complication associated with poor prognosis in patients with acute coronary syndrome. ${ }^{39}$ Several studies examined the characteristics of the culprit lesion in ST-elevation myocardial infarction and the role of plaque volume in the pathogenesis of slow flow, and found that the presence of IVUS parameters (lesion of the external elastic membrane at the cross-section area, plaque area, plaque volume, and remodeling index) was significantly higher in the slow flow group compared to the normal flow group. ${ }^{15,40-42}$

IVUS can also be used for evaluation before and after stent placement, and to identify the presence of in-stent restenosis. A recent study demonstrated the importance of assessing the severity of remodeling using IVUS, indicating that an IVUS-guided strategy is more effective for stent implantation than angiography alone. IVUS-guided procedures were associated with significantly higher rates of optimal stent expansion and decreased risk of stent underexpansion, malposition, and residual stenosis. ${ }^{13}$

IVUS is an essential clinical method for studying arterial remodeling in vivo ${ }^{43}$; however, this is not relevant to the use of IVUS in daily clinical practice because IVUS analysis of three vessels is more time consuming and may increase the risk of complications. ${ }^{38}$

\section{Optical Coherence Tomography}

The use of optical coherence tomography (OCT) is becoming increasingly important in the investigation of coronary artery disease. OCT offers high-resolution images that re- 
veal the nature and composition of the plaque. We usually draw a parallel between IVUS and OCT, but in reality, they use two different technologies to produce intracoronary images. Both investigations have their advantages and disadvantages and should be seen as complementary to each other. The axial resolution of OCT is better, superior for the examination of calcified plaques, visualization of fibrosus cap and intima morphology, and detection of macrophages. In contrast, IVUS has a higher tissue penetration and is better in identifying plaque burden and evaluating vascular remodeling, but it lacks the spatial resolution needed for small features, for example to identify details of the intima and biochemical changes associated with the formation of an atherosclerotic plaque. ${ }^{44-46}$

\section{THE IMPACT OF TYPE 2 DIABETES ON CORONARY ARTERY REMODELING}

Several studies have addressed the association between negative coronary artery remodeling and type 2 diabetes. ${ }^{47}$ Jensen et al. showed that in patients with associated diabetes, compensatory vessel enlargement is less common, in contrast to coronary artery narrowing. ${ }^{48}$ The study also showed that the presence of diabetes was associated with greater atherosclerotic progression and damage to the compensatory remodeling of the artery wall. Also, inadequate compensatory remodeling was more evident in insulin-treated patients. As the vessel wall reacts negatively to insulin, the proliferation of smooth muscle and fibrous tissue increases wall stiffness, thereby impairing its compensatory expansion capacity. ${ }^{49,50}$ However, increased insulin resistance was significantly associated with a higher remodeling index and positive coronary artery remodeling. ${ }^{51}$ Serum levels of glycated albumin were elevated but serum endogenous secretory receptor for advanced glycation end-products (esRAGE) levels were decreased in diabetic patients with negative remodeling. ${ }^{50}$ A recent study published in 2020 showed that some of the glucose-related variables, such as HbA1c, are significantly and positively associated with negative coronary artery remodeling. ${ }^{52}$

\section{CONCLUSION}

Excluding the presence of the coronary heart disease or determining the extent of disease plays an important role in the study of patients with chest pain. As a noninvasive imaging method, coronary computed tomography angiography is able to provide accurate data of the coronary artery wall and to assess the presence and components of an atherosclerotic plaque, even in non-flow-limiting coronary artery disease. Over the next few years, the number of coronary computed tomography angiography examinations is likely to increase significantly, and its role as a "gatekeeper" prior to invasive angiography will be further strengthened. To increase diagnostic accuracy, invasive coronary angiography can be associated with intravascular ultrasound and optical coherence tomography.

\section{CONFLICT OF INTEREST}

Nothing to declare.

\section{ACKNOWLEDGEMENT}

This research was supported via the research grant no. 103544/2016, contract number 26/01.09.2016, entitled "Increasing the research capacity in the field of vulnerable plaque imaging, based on advanced nanoparticles, fusion imaging and computational simulation - PlaqueImage", financed by the Romanian Ministry of European Funds, the Romanian Government and the European Union.

\section{REFERENCES}

1. Virmani R, Burke AP, Farb A, Kolodgie FD. Pathology of the vulnerable plaque. J Am Coll Cardiol. 2006;47:C13-18

2. Overbaugh KJ. Acute Coronary Syndrome. AJN Am J Nurs. 2009;109:42-52

3. Clarke J-RD, Duarte Lau F, Zarich SW. Determining the Significance of Coronary Plaque Lesions: Physiological Stenosis Severity and Plaque Characteristics. J Clin Med. 2020;9:665.

4. Stefanadis C, Antoniou C, Tsiachris D, Pietri P. Coronary Atherosclerotic Vulnerable Plaque: Current Perspectives. J Am Heart Assoc. 2017;6 e005543

5. Drobni ZD, Kolossváry M, Szilveszter B, Merkely B, Maurovich-Horvat P. A koronária-CT-angiográfia jelentősége a mindennapi gyakorlatban stabil anginás betegek körében. Cardiol Hung. 2018;48:52-57.

6. Crawford T, Levene $\mathrm{Cl}$. Medial thinning in atheroma. J Pathol Bacteriol. 1953;66:19-23.

7. Glagov S, Weisenberg E, Zarins CK, Stankunavicius R, Kolettis GJ. Compensatory Enlargement of Human Atherosclerotic Coronary Arteries. N Engl J Med. 1987;316:1371-1375.

8. Hermiller JB, Tenaglia AN, Kisslo KB, et al. In vivo validation of compensatory enlargement of atherosclerotic coronary arteries. Am J Cardiol. 1993;71:665-668.

9. Varnava AM, Mills PG, Davies MJ. Relationship Between Coronary Artery Remodeling and Plaque Vulnerability. Circulation. 2002;105:939-943.

10. Schoenhagen P, Ziada KM, Kapadia SR, Crowe TD, Nissen SE, Tuzcu EM. Extent and Direction of Arterial Remodeling in Stable Versus Unstable Coronary Syndromes: An Intravascular Ultrasound Study. Circulation. 2000;101:598-603.

11. Nishioka T, Luo H, Eigler NL, Berglund H, Kim C-J, Siegel RJ. Contribution of inadequate compensatory enlargement to development of human coronary artery stenosis: An in vivo intravascular ultrasound study. J Am Coll Cardiol. 1996;27:1571-1576.

12. Mintz GS, Kent KM, Pichard AD, Satler LF, Popma JJ, Leon MB. Contribution of Inadequate Arterial Remodeling to the Development of Focal Coronary Artery Stenoses: An Intravascular Ultrasound Study. Circulation. 1997;95:1791-1798.

13. Hakim D, Abdallah M, Effat M, Al Solaiman F, Alli O, Leesar MA. A new intravascular ultrasound-guided stenting strategy compared with angiography on stent expansion and procedural outcomes in patients 
with positive lesion remodeling. Catheter Cardiovasc Interv. 2021;97:237244.

14. Galal H, Rashid T, Alghonaimy W, Kamal D. Detection of positively remodeled coronary artery lesions by multislice $\mathrm{CT}$ and its impact on cardiovascular future events. Egypt Heart J. 2019;71:26.

15. Reddy S, Rao K R, Kashyap JR, et al. Impact of plaque burden and composition on coronary slow flow in ST-segment elevation myocardial infarction undergoing percutaneous coronary intervention: intravascular ultrasound and virtual histology analysis. Acta Cardiol. 2020;1-11.

16. Rodriguez-Granillo GA. Coronary artery remodelling is related to plaque composition. Heart. 2005;92:388-391.

17. Inaba S, Mintz GS, Farhat NZ, et al. Impact of Positive and Negative Lesion Site Remodeling on Clinical Outcomes. JACC Cardiovasc Imaging. 2014;7:70-78

18. Gussenhoven EJ, Geselschap JH, van Lankeren W, Posthuma DJ, van der Lugt A. Remodeling of Atherosclerotic Coronary Arteries Assessed With Intravascular Ultrasound In Vitro. Am J Cardiol. 1997;79:699-702.

19. Nakamura M, Nishikawa $\mathrm{H}$, Mukai S, et al. Impact of coronary artery remodeling on clinical presentation of coronary artery disease: an intravascular ultrasound study. J Am Coll Cardiol. 2001;37:63-69.

20. Eckert J, Schmidt M, Magedanz A, Voigtländer T, Schmermund A. Coronary CT Angiography in Managing Atherosclerosis. Int J Mol Sci. 2015;16:37403756.

21. Virmani R, Burke AP, Farb A, Kolodgie FD. Pathology of the Vulnerable Plaque. J Am Coll Cardiol. 2006;47:C13-C18.

22. Burke AP, Farb A, Malcom GT, Liang YH, Smialek J, Virmani R. Coronary risk factors and plaque morphology in men with coronary disease who died suddenly. N Engl J Med. 1997;336:1276-1282.

23. Gueret P, Deux J-F, Bonello L, et al. Diagnostic Performance of Computed Tomography Coronary Angiography (from the Prospective National Multicenter Multivendor EVASCAN Study). Am J Cardiol. 2013:111:471-478.

24. Knuuti J, Wijns W, Saraste A, et al. 2019 ESC Guidelines for the diagnosis and management of chronic coronary syndromes. Eur Heart J. 2020;41:407-477.

25. The SCOT-HEART Investigators. Coronary CT Angiography and 5-Year Risk of Myocardial Infarction. N Engl J Med. 2018;379:924-933.

26. Hoffmann U, Ferencik M, Udelson JE, et al. Prognostic Value of Noninvasive Cardiovascular Testing in Patients With Stable Chest Pain: Insights From the PROMISE Trial (Prospective Multicenter Imaging Study for Evaluation of Chest Pain). Circulation. 2017;135:2320-2332.

27. Mark DB, Shaw L, Harrell FE, et al. Prognostic Value of a Treadmill Exercise Score in Outpatients with Suspected Coronary Artery Disease. N Engl J Med. 1991;325:849-853.

28. Motoyama S, Sarai M, Harigaya H, et al. Computed Tomographic Angiography Characteristics of Atherosclerotic Plaques Subsequently Resulting in Acute Coronary Syndrome. J Am Coll Cardiol. 2009;54:49-57.

29. Conte E, Annoni A, Pontone G, et al. Evaluation of coronary plaque characteristics with coronary computed tomography angiography in patients with non-obstructive coronary artery disease: a long-term followup study. Eur Heart J - Cardiovasc Imaging. 2017;18:1170-1178.

30. Motoyama S, Ito H, Sarai M, et al. Plaque Characterization by Coronary Computed Tomography Angiography and the Likelihood of Acute Coronary Events in Mid-Term Follow-Up. J Am Coll Cardiol. 2015;66:337346

31. Han D, Berman DS, Miller RJH, et al. Association of Cardiovascular Disease Risk Factor Burden With Progression of Coronary Atherosclerosis Assessed by Serial Coronary Computed Tomographic Angiography. JAMA Netw Open. 2020;3:e2011444.

32. Williams MC, Moss AJ, Dweck M, et al. Coronary Artery Plaque Characteristics Associated With Adverse Outcomes in the SCOT-HEART Study. J Am Coll Cardiol. 2019;73:291-301.

33. Nazir MS, Nicol E. The SCOT-HEART trial: cardiac CT to guide patient management and improve outcomes. Cardiovasc Res. 2019;115:e88-e90.

34. Schoenhagen P, Ziada KM, Vince DG, Nissen SE, Tuzcu EM. Arterial remodeling and coronary artery disease: the concept of "dilated" versus "obstructive" coronary atherosclerosis. J Am Coll Cardiol. 2001;38:297306.

35. Serruys PW, Katagiri $Y$, Sotomi $Y$, et al. Arterial Remodeling After Bioresorbable Scaffolds and Metallic Stents. I Am Coll Cardiol. 2017;70:60-74

36. Ono M, Kawashima $\mathrm{H}$, Hara $\mathrm{H}$, et al. Advances in IVUS/OCT and Future Clinical Perspective of Novel Hybrid Catheter System in Coronary Imaging. Front Cardiovasc Med. 2020;7:119.

37. de Boer S, Baran Y, Garcia-Garcia HM, et al. The European Collaborative Project on Inflammation and Vascular Wall Remodeling in Atherosclerosis - Intravascular Ultrasound (ATHEROREMO-IVUS) study. Eurolntervention. 2018;14:194-203.

38. Cheng JM, Garcia-Garcia HM, de Boer SPM, et al. In vivo detection of high-risk coronary plaques by radiofrequency intravascular ultrasound and cardiovascular outcome: results of the ATHEROREMO-IVUS study. Eur Heart J. 2014;35:639-647.

39. Yamamoto K, Sakakura K, Akashi N, et al. Association of slow flow with clinical factors in intravascular ultrasound-guided percutaneous coronary intervention for patients with left main trunk-acute myocardial infarction. $J$ Cardiol. 2020;75:53-59.

40. Hong YJ, Jeong MH, Choi YH, et al. Positive remodeling is associated with more plaque vulnerability and higher frequency of plaque prolapse accompanied with post-procedural cardiac enzyme elevation compared with intermediate/negative remodeling in patients with acute myocardial infarction. J Cardiol. 2009;53:278-287.

41. Nakamura T, Kubo N, Ako J, Momomura S-I. Angiographic No-Reflow Phenomenon and Plaque Characteristics by Virtual Histology Intravascular Ultrasound in Patients with Acute Myocardial Infarction. J Intervent Cardiol. 2007:20:335-339.

42. Li J, Wu L, Tian X, Zhang J, Shi Y. Intravascular Ultrasound Observation of the Mechanism of No-Reflow Phenomenon in Acute Myocardial Infarction. Zhang Z, ed. PLoS One. 2015;10:e0119223.

43. Nissen SE, Gurley JC, Grines CL, et al. Intravascular ultrasound assessment of lumen size and wall morphology in normal subjects and patients with coronary artery disease. Circulation. 1991;84:1087-1099.

44. Ali ZA, Maehara A, Généreux P, et al. Optical coherence tomography compared with intravascular ultrasound and with angiography to guide coronary stent implantation (ILUMIEN III: OPTIMIZE PCI): a randomised controlled trial. The Lancet. 2016;388:2618-2628.

45. Xu J, Lo S. Fundamentals and role of intravascular ultrasound in percutaneous coronary intervention. Cardiovasc Diagn Ther. 2020;10:1358-1370.

46. Bec J, Phipps JE, Gorpas D, et al. In vivo label-free structural and biochemical imaging of coronary arteries using an integrated ultrasound and multispectral fluorescence lifetime catheter system. Sci Rep. 2017;7:8960.

47. Reddy S, Kadiyala V, Kashyap JR, et al. Comparison of Intravascular Ultrasound Virtual Histology Parameters in Diabetes versus Non-Diabetes with Acute Coronary Syndrome. Cardiology. 2020;145:570-577.

48. Jensen LO, Thayssen P, Mintz GS, et al. Intravascular ultrasound assessment of remodelling and reference segment plaque burden in type-2 diabetic patients. Eur Heart J. 2007;28:1759-1764.

49. Nicholls SJ, Tuzcu EM, Kalidindi S, et al. Effect of Diabetes on Progression of Coronary Atherosclerosis and Arterial Remodeling. J Am Coll Cardiol. 2008:52:255-262.

50. Du R, Zhang RY, Lu L, et al. Increased glycated albumin and decreased esRAGE levels in serum are related to negative coronary artery remodeling in patients with type 2 diabetes: an Intravascular ultrasound study. Cardiovasc Diabetol. 2018:17:149.

51. Kim S-H, Moon J-Y, Lim YM, et al. Association of insulin resistance and coronary artery remodeling: an intravascular ultrasound study. Cardiovasc Diabetol. 2015;14:74.

52. Liu J, Wang S, Cui C, et al. The association between glucose-related variables and plaque morphology in patients with ST-segment elevated myocardial infarction. Cardiovasc Diabetol. 2020;19:109. 